\title{
A Qualitative Assessment of People-Centeredness of Inpatient Tuberculosis Treatment Services in Armenia
}

\author{
Zaruhi Grigoryan*, Nune Truzyan, Lusine Musheghyan, Varduhi Petrosyan \\ Avedisian Onanian Center for Health Services Research and Development, Turpanjian School of Public Health, American \\ University of Armenia, 40 Marshal Baghramyan, Yerevan, Republic of Armenia \\ Email: `zgrigoryan@aua.am, truzyan.nune@gmail.com,lmusheghyan@aua.am,vpetrosi@aua.am
}

How to cite this paper: Grigoryan, Z., Truzyan, N., Musheghyan, L. and Petrosyan, V. (2021) A Qualitative Assessment of PeopleCenteredness of Inpatient Tuberculosis Treatment Services in Armenia. Journal of $\mathrm{Tu}$ berculosis Research, 9, 184-196. https://doi.org/10.4236/jtr.2021.93017

Received: June 3, 2021

Accepted: August 13, 2021

Published: August 16, 2021

Copyright $\odot 2021$ by author(s) and Scientific Research Publishing Inc. This work is licensed under the Creative Commons Attribution International License (CC BY 4.0).

http://creativecommons.org/licenses/by/4.0/

(c) (i) Open Access

\begin{abstract}
Background: People-centered tuberculosis (TB) care promotes treatment adherence and outcomes. TB patients' and families' health education and protection of their rights are among the core components of people-centered care. We aimed to assess the level of people-centeredness of TB care as a proxy to quality in the largest inpatient unit of the National Pulmonology Center (NPC) in Armenia. Methods: We conducted a qualitative study by interviewing clinical and administrative staff, TB patients, and family members to learn their experiences about patient and family education and rights (PFE\&R) protection practices focusing on two Joint Commission International (JCI) Standards for Hospital Accreditation. Mixed-conventional inductive and directed deductive content approach guided the analysis of data. Results: The study revealed various gaps in the provided services. According to the TB physicians and nurses, they routinely educated patients and families and took actions to protect their rights. However, practices reported by TB providers varied across clinical departments and professionals and did not meet the recommendations of the JCI standards. The document review revealed that no written policies or procedures existed in the NPC inpatient unit to guide the implementation of PFE\&R. Lastly, patients' inconsistent experiences were also indicative of the lack of standardization and issues with PFE\&R implementation. Conclusion: Bridging the gap between existing and recommended practices by establishing and enforcing new people-centered policies and procedures is a pledge for improving operations and patients' experiences with a potential nationwide impact in Armenia.
\end{abstract}

\section{Keywords}

Patient Education, Patient Rights, Quality Assessment 


\section{Introduction}

Tuberculosis (TB) is a major global public health concern around the world [1]. Between 2000 and 2017, 54 million deaths have been averted due to successful global strategies; nevertheless, in 2019, TB was still responsible for 1.4 million deaths worldwide [1]. In 2018, the TB incidence rate in Armenia was 31 per 100,000 population, with a 1.3 mortality rate per 100,000 [2]. Though the overall incidence of $\mathrm{TB}$ has been falling during recent years in Armenia, the emergence and progression of drug-resistant TB (DR-TB) continue to threaten the national TB control efforts [3]. Despite freely available and accessible TB diagnostic and treatment services throughout the country, non-adherence to TB treatment remains a major challenge for TB control in Armenia [3].

High-quality TB care is an important step toward improving medical practice and patient outcomes, therefore decreasing the TB burden [4] [5]. The International Standards for TB Care (ISTC) have set a quality benchmark for national TB programs to facilitate provision of high-quality care to patients [5] [6]. Quality improvement within TB care is possible once diagnostic and treatment services satisfy international standards adapted for the countries' needs and supported by the development of quality improvement programs [6].

An intrinsic component of quality of care is patient-centeredness, which is effective in promoting TB adherence, treatment outcomes, and care for TB, including multi-drug resistant TB (MDR-TB), provision of psychological support, and overcoming social stigma [7]-[13]. Indeed, the goal of the ninth ISTC standard is provision of patient-centered TB care as a means for promoting TB treatment adherence, improved quality of life, and relief from suffering [5]. In response to the emerging evidence, patient-centered integrated $\mathrm{TB}$ care has been incorporated as a core pillar of the End-TB strategy [8]. During recent years, the concept of patientcentered TB care has evolved into a more holistic people-centered model of care. Though the terms are yet used interchangeably, the people-centered model considers all health, societal and economic concerns of a healthcare recipient, going beyond the label of a "patient". Here, the person is viewed within the trajectory of his/her environment, i.e. family and community who are immediately involved in the prevention, progression, and treatment of TB [14] [15] [16] [17]. In general, integrated people-centered care assumes that the core of health systems is people and communities rather than diseases. The new model of care aims to empower communities to take a lead of building their own health, instead of being inactive recipients [18]. Meanwhile, integrated care implies that effective healthcare systems surround the communities, to support the continuity of health needs [18].

Concordant with global targets, healthcare facilities are supposed to identify, establish, and maintain an environment where patients' and families' needs are respected [19]. Patients' and families' awareness-raising, especially in long-term therapies, is essential for improving treatment adherence and ultimately achieving positive treatment outcomes [19] [20] [21]. According to the Joint Commis- 
sion International (JCI) Standards for Hospital Accreditation, actions towards improving the health knowledge of patients and families should start from educational needs assessments. The latter should expand to a hands-on training on treatment process, provision of complete information on the disease, and guidance in the decision-making process [19].

In addition, local quality improvement strategies should necessarily incorporate promotion of patients' and families' rights [22]. In order to promote patients' autonomy, adherence to treatment, and facilitation of trust in the healthcare system, TB patients and families should have access to complete information on rights and responsibilities and TB disease and its health impact [23]. Healthcare systems should set policies and regulations defining patients' rights, healthcare providers' and facilities' responsibilities in maintaining them along with introducing enforcement mechanisms [24] [25]. To satisfy the JCI standards on patient and family rights, healthcare facilities and clinical staff should recognize those rights and act accordingly to protect and promote them. Defining the scope of patients' and families' involvement in care-related decisions and an informed consent process are essential components of patients' right protection and promotion [19].

We aimed to assess the level of people-centeredness in the largest inpatient TB facility of the National Pulmonology Center (NPC) in Armenia and evaluate how healthcare providers value, maintain and satisfy patients' and their families' rights and needs for health education during inpatient treatment. Therefore, taking two JCI quality standards as a quality benchmark, we qualitatively assessed the key informants' experiences and practices on patient and family education and rights protection.

\section{Methods}

\subsection{Study Design and Setting}

This assessment was conducted within the scope of the bigger project Quality of Inpatient Tuberculosis Health Care that explored the needs of TB diagnostic and treatment services in the TB inpatient unit of the NPC located in Abovian city, Armenia [26]. The TB inpatient unit has 300 beds and serves almost $90 \%$ of all inpatient TB and MDR-TB cases countrywide.

To explore the quality of patients' and families' education and their rights protection processes we qualitatively assessed TB inpatient unit's clinical and administrative staff practices as well as patients' experiences against the patient and family rights and education-related JCI standards through in-depth interviews (IDI) using semi-structured interview guides. Utilization of qualitative research methods for assessing quality of care helped to gain a deeper picture of service quality reflecting the in-depth experiences and perspectives of various parties [27]. We used two people-centered JCI functions-Patient and Family Education (PFE) and Patient and Family Rights (PFR) (PFE \& R) as a framework for the assessment. Overall, six PFE and nine PFR standards along with their measurement criteria- 
measurable elements (MEs) $(n=48)$ guided the development of IDI guides and the whole analysis.

\subsection{Study Participants}

TB healthcare providers (HCPs) (heads of departments, TB physicians, and nurses) and administrative staff were purposively recruited in the study. To increase variability in responses and gain a complete understanding about the level of peoplecenteredness in the TB inpatient unit, we approached HCPs from all clinical departments (drug-sensitive TB, drug-resistant $\mathrm{TB}$, pediatric $\mathrm{TB}$, extra-pulmonary TB) of the NPC. To gain a comprehensive picture on quality of PFE\&R we also explored the recipients' perspectives by inviting to participate in the study those patients and family members, who received their intensive phase of treatment in the NPC inpatient unit. The inclusion criterion for HCPs was being employed in the clinical departments of the TB inpatient unit, and the exclusion criterion was working as a narrow specialist (cardiologist, endocrinologist, gynecologist, etc.). The inclusion criteria for patients were having a history of inpatient treatment in the TB inpatient unit and receiving outpatient phase of the treatment at the time of the study. The general inclusion criteria applied to all study participants were having the ability to understand and speak Armenian, and giving verbal consent to participate.

\subsection{Study Instruments}

Based on the JCI PFE\&R standards, specific requirements to proper PFE\&R practices represented by the MEs were reformulated either into open-ended questions to explore them qualitatively or into checklists to guide the document review. Table 1 presents the standards used for developing the overall content of the assessment and the study instruments. Eventually, three semi-structured interview guides were developed for moderating IDIs with heads of clinical departments, TB physicians/nurses, and patients/family members. We also developed two checklists for the review of legal/policy documents and medical records [26].

\subsection{Data Collection and Analysis}

The data was collected during March-May 2016. Overall, we conducted 26 IDIs with $21 \mathrm{HCPs}$, four patients and a family member. The average duration of the interviews was 40 minutes. All IDIs were held in the TB inpatient unit. To prevent discomfort and facilitate honest responses we avoided audio-recording of the interviews, instead, the researchers took detailed notes. All participants provided verbal consent to participate in the study. Detailed notes taken during interviews were immediately transcribed. We stopped data collection when saturation was achieved. We also reviewed 10 legal documents, including policies and regulations of the TB inpatient unit, and 34 medical records to complete the checklists. We conducted coding and grouping of repeating phrases and sentences in accordance with the predefined MEs of the standards under two major 
Table 1. Six Patient and Family Education and Nine Patient and Family Rights Standards.

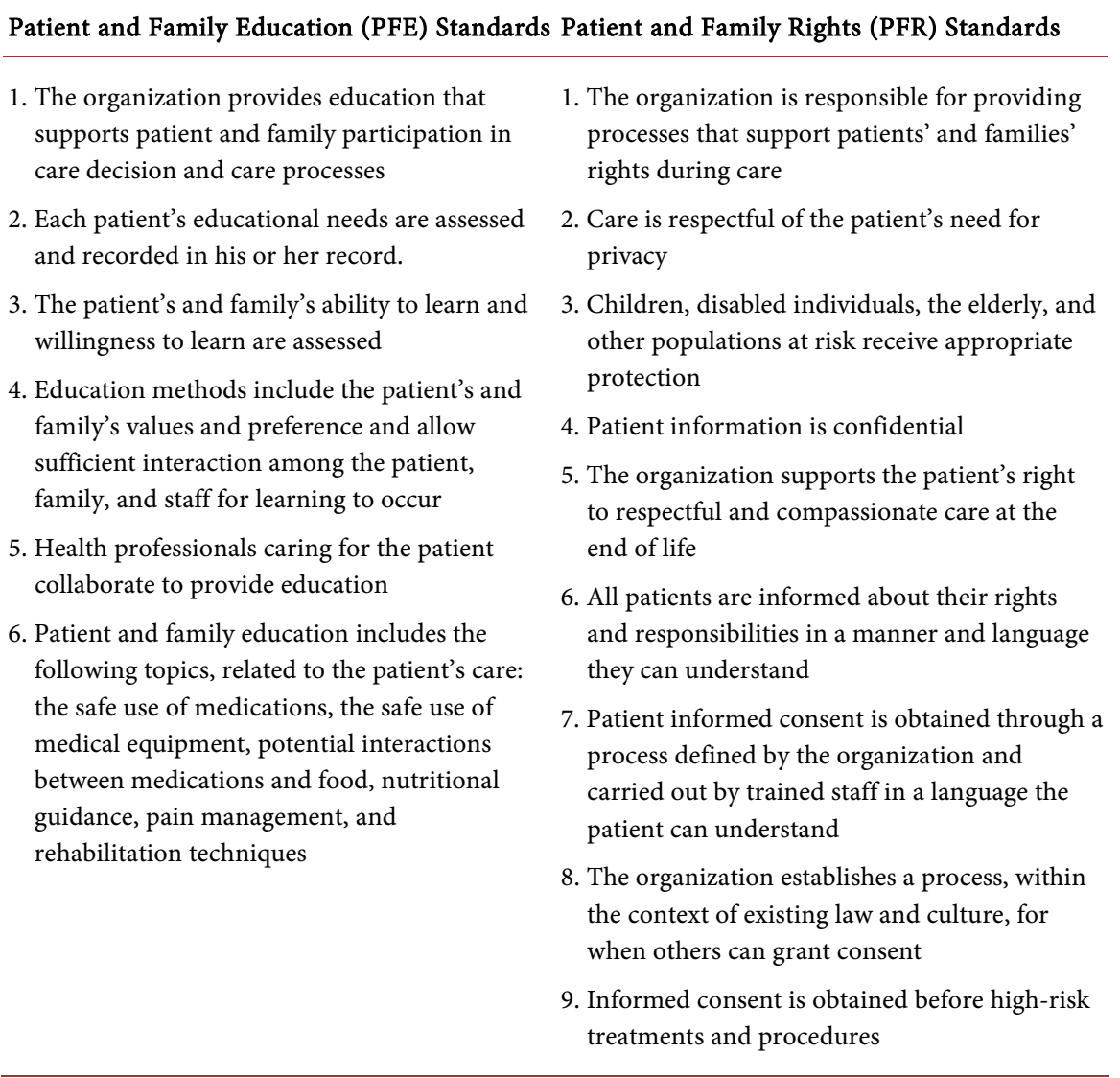

themes: PFE and PFR. We used mixed conventional inductive and directed deductive content analysis to explore the data [28] [29].

\subsection{Study Rigor}

Two researchers, who have been extensively trained in qualitative research methods and study protocol, collected and analyzed the data. They built rapport with HCPs and patients. While conducting the IDIs, the researchers rotated the roles of the facilitator and note-taker to minimize variability between interviewers. To increase the trustworthiness of the results, we used standard qualitative research techniques of heterogeneity and triangulation [30]. Triangulation within several groups of respondents and through different data collection techniques increased the reliability of the results.

\subsection{Study Ethics}

The Institutional Review Board (IRB) of the American University of Armenia approved the study protocol (\#AUA-2016-002).

\section{Results}

The section presents the main findings of the interviews with study participants. HPCs shared their experiences on how they act to improve patients' and fami- 
lies' health literacy and knowledge about TB and protect patients' and families' rights, their needs for privacy, and confidentiality. We summarized the main findings into two main themes.

\subsection{Patient and Family Education}

PFE was not uniformly organized in the TB inpatient unit, as there were missing policies and procedures on improving patients' and families' TB knowledge and the formal educational program. HCPs of the TB inpatient unit did not share a common standpoint about how and who should organize patient and family education during inpatient treatment. Some participants, such as heads of departments and nurses were certain that patient and family education on topics related to TB was the inpatient HCPs' immediate function. However, others noted that the primary HCPs were responsible for improving awareness about TB and not the inpatient physicians.

The patients' education starts in hospital admission. We notify what is going to happen to them in the hospital while trying to make the patients feel positive about the treatment. (Head of department)

It is the nurses' responsibility to lead educational sessions with patients. (Nurse)

The educational activities with patients should not be done in the hospital. We are not supposed to educate families. We may just talk to them if needed, but education is not part of our responsibilities. We do not put people in a line in the corridor to provide a lecture [on TB-related topics]. (Head of department)

In addition, some physicians complained about the scarcity of time, which, in fact, was the most commonly cited reason for not providing formal and uniform education to all patients. According to them, the detailed counseling sessions focusing on health education about the disease and treatment were needless, given the limited time.

We have 10 - 15 patients and a healthcare provider does not have enough time to educate all patients with all details. We are overloaded with paperwork...Some patients ask for very detailed questions. We cannot explain the whole treatment to them. There is no need to talk about too detailed things; it is time-consuming. (Physician)

During the interviews, physicians blamed patients' educational level as one of the reasons for having poor awareness of TB.

People are different. If one is adequate and smart enough, we explain to him/her and the family that before sputum conversion they should try to avoid kissing each other. Much depends on the educational level of patients... (Physician)

The IDIs and medical record reviews revealed that during inpatient treatment, HCPs neither assessed TB patients' educational needs and willingness to learn nor documented any relevant information. Furthermore, they men- 
tioned that topics to be discussed during the counseling sessions depended on patients' needs. Both patients and physicians were consistent that whenever patients and family members asked questions, they exhaustively answered them. Former TB patients described some topics that were discussed. There is no specific list of topics to educate patients. It depends on the patients' needs. (Physician)

Whenever the patient asks questions, we answer. (Physician)

I was told, that TB is curable and I should not be afraid of it... If something was not clear, I was asking and clarifying with the physicians. I was told that if I took the medications, did not interrupt the treatment, eat good enough, the disease would leave... They also told me that $80 \%$ of treatment success depended on me. I was also told that if I did not take medication as prescribed and if I stopped taking them when feeling better, the disease could become untreatable. (Patient/Family member)

They [HCPs] told me that I had drug-sensitive TB and that it was curable. They [HCPs] also told me what I should expect after the treatment. I did not have unanswered questions. (Patient/Family member)

Physicians mentioned that the information provided to patients was not supported with informational materials, though they considered those materials useful.

Sometimes, educational materials need to be provided to patients [the TB inpatient unit does not have educational materials for patients]. (Physician) According to the majority of HCPs, they did not specifically encourage patients to be active and ask questions and did not verify if patients understood the provided information. Instead, physicians assessed patients' awareness on topics related to their disease and treatment indirectly, during their random conversations.

If they [patients and families] ask [questions], we respond to them, but not the opposite. (Physician)

Our patients stay here for a long period. We inform them [about the disease] and later during the conversations it becomes clear whether they have understood us or not. (Physician)

Despite the absence of uniform practices to improve patients' and families' awareness of TB, the majority of patients valued HCPs' professional competencies mentioning that those who educated patients and families were characterized as knowledgeable individuals with good communication skills. Patients further elaborated that physicians and nurses worked collaboratively to meet their need for information.

I am satisfied with their competencies and attitude. (Patient/Family member)

The professionals [healthcare providers] were very good. (Patient/Family member)

Nurses and physicians informed us [about TB] so that we know everything and are informed. (Patient/Family member) 


\subsection{Patient and Family Rights}

The study team found that there were no policies or procedures guiding PFR in the TB inpatient unit. In fact, no specific actions were implemented by the leadership to convey the vision of PFR protection to the clinical staff. The staff failed to identify the roles of HCPs and healthcare leaders in protecting TB patients' rights. Despite the absence of formal paperwork, nearly all HCPs highlighted their respect towards patient rights and towards patients' prerogative to choose what information should be provided to families and others. However, during the counseling, physicians reportedly did not assess patients' expectations and needs for privacy. Yet, the majority of them appeared to be respecting patients' needs for privacy by communicating with them privately. In contrast to this, a few patients reported that their counseling was organized in the presence of other patients.

The counseling was done in my room, in the presence of another patient. The psychologist came for that patient and also offered services to me, but I did not feel comfortable during the whole process, thus, I was not satisfied. (Patient/Family member)

The majority of physicians did not know any hospital-specific definition of vulnerable patient groups and did not even know if such groups existed. Most of the time, health providers mistakenly mentioned co-morbidities known to influence TB treatment outcomes as indicators of vulnerability. The vulnerable groups include patients with diabetes, AIDS, ulcer disease, professional conditions [occupational diseases] .... Children and those who have a TB contact in their household are also vulnerable groups. (Physician) Patients reported that they were told that their personal and health information would be kept confidential and would not be communicated to other people. Some of the clinical staff members identified the notion of confidentiality assurance only as confidentiality protection for HIV/AIDs patients. However, some of the physicians appeared to be not fully practicing confidentiality protection in terms of informing patients about how their personal and health-related information would be protected.

I was told, that everything [health-related and personal information] is confidential and if I want to keep my disease in secret, no one will know about it. (Patient/Family member)

We are especially cautious when working with HIV/AIDS patients. We try to maintain $100 \%$ confidentiality, knowing that we should not speak about that disease [HIV/AIDS]. (Healthcare provider)

I did not have issues with confidentiality. But no one has told me that the information related to me and my health will be kept confidential. (Patient/ Family member)

Physicians expressed strong empathy towards the patients receiving end-oflife care and mentioned that they worked to protect patients from physical or emotional pain. 
This [the end of life care] is a painful topic. We [the clinical staff] surely notify the family members about the severity of the patient's condition and inform them about the possible outcomes. We can never tell that the patient is going to die, and we should not talk to patients about such issues. Perhaps from the psychological perspective, it is wrong, but we try to cheer the patient up by all means we have. (Physician)

We found that only in the DR-TB department patients sign a contract for treatment that includes information on their rights and responsibilities. The process has been established by the MSF France that took the responsibility of coordinating the DR-TB care in the inpatient unit. TB patients from other departments did not formally receive such information. Besides this informal process, the document review did not identify any policy guiding the content and the process of informed consent, and no specific staff members were assigned to implement the informed consent in the TB inpatient unit. In addition, no formal processes were established to obtain an informed consent from people other than patients in case if they needed emergency high-risk procedures.

\section{Discussion}

This is the first study that assessed the level of people-centeredness of inpatient TB care in Armenia by applying JCI Hospital Accreditation Standards focused on the best recommended practices related to patient and family education and their rights protection.

The TB inpatient unit did not introduce and enforce policies and procedures guiding the processes of protecting PFR and providing PFE. According to Epstein et al., improvement of communication between HCPs, patients, and families can be possible through the development of written policies that guide the healthcare processes [31].

TB HCPs expressed controversial attitudes towards TB patients' awarenessraising, as some of them agreed that patients should be educated on TB and TB care, yet some physicians disputed whether this was the responsibility of inpatient TB physicians.

Though patients were not receiving a structured and formal education, TB physicians gave some information to patients. We learned that the process of improving patients' knowledge about $\mathrm{TB}$ was patient-driven as physicians were inclined to wait for patients to ask questions instead of initiating educational discussions. Nevertheless, patients seemed to be satisfied with whatever information they received. Harutyunyan et al. explained the impact of low expectations from HCPs on high level of satisfaction from low quality services among the Armenian population [32]. In fact, the absence of educational sessions and the patient-driven education are more indicative of the poor quality of services as the content of the information provided to patients was not standardized and varied across HCPs and patients. The literature suggests that adequate knowledge about TB has a protective effect against possible non-adherence [33]. Furthermore, Mo- 
risky et al. suggested that a structured education program could improve the continuity of care and improve adherence to TB treatment [34].

Our findings were controversial on privacy protection as HCPs claimed that patients' privacy was respected, whereas some patients shared their negative experiences when personal information was communicated to them in the presence of a third party. Though HCPs acknowledged that confidentiality protection was an intrinsic part of medical ethics, overall the TB inpatient unit did not perform well on this matter. Patients did not share any bad experiences regarding their confidentiality protection since the majority of them were not informed about how and what information would be kept confidential and what laws and regulations underpinned confidentiality of patient information. Patients and families would not be able to recognize any violation, since they did not know what to expect from the process of care. The HCPs' practices on confidentiality assurance were based on their own perceptions and understanding, therefore seemed to vary between professionals. Patient rights promotion is known to be essential from the perspective of quality [22]. Furthermore, the WHO states that maintenance of privacy and confidentiality of TB patients' health information is essential for tackling TB associated stigma and establishing trust between the patient and the communities [23].

The comparison of participants' reflections with the JCI-recommended practices revealed a poor degree of people-centeredness of inpatient TB services implying about the compromised quality of care. Given that the patient-centered care has been widely acknowledged as a pledge for improved TB care, adherence and outcomes and that the concept has further evolved to encompass also the societal aspects of disease development and progression, it was of utmost importance to promote establishment and provision of people-centered TB care in the largest inpatient TB unit in Armenia with a potential of nationwide impact on the disease burden.

In fact, the study findings contributed to the policy change in the country as starting 2019, to address the identified issues regarding the quality of care, Armenia introduced the people-centered model of care supported by the updated National TB Treatment Guideline [16] [35]. Currently, at the beginning of the intensive phase of treatment, patients receive educational and psychological counseling, and supportive family members assist patients throughout the continuation phase of the treatment to facilitate better treatment adherence and outcomes [16].

Though assessment of staff performance might lead to providers' exaggeration of their actual practices regarding PFE \& R, resulting in demonstration of slightly better practices than the reality, the findings of this study might be of significant relevance to other low- and middle-income countries experiencing similar TB burden with a similar model of patient-provider interaction. The quality assessment tools can also be used for similar assessments in outpatient TB units. Our study methodology and findings might be of high interest for healthcare systems 
aiming to establish people-centered TB treatment services.

\section{Conclusion}

Bridging the gap between the existing and recommended evidence-based practices was a pledge for promoting people-centered TB care approaches in the NPC inpatient unit in Armenia. The study findings can be used for designing interventions targeted at assessing and improving quality of care in TB inpatient and outpatient units elsewhere specifically focusing on the protection of PFE\&R. In addition, the described methodology of assessing people-centeredness of care can be successfully extrapolated to other health contexts.

\section{Acknowledgements}

We are thankful to the administration and clinical staff of the National Pulmonology Center for their continuous support throughout the study.

\section{Funding}

The work was financially supported by the Armenian Medical Fund, United States.

\section{Authors' Contributions}

NT, ZG, LM, and VP designed the study. NT, ZG, and LM conducted the study. ZG and NT drafted the paper. ZG, NT, LM and VP reviewed and approved the paper for publication.

\section{Conflicts of Interest}

The authors declare no conflicts of interest regarding the publication of this paper.

\section{References}

[1] World Health Organization (2020) Global Tuberculosis Report 2020. World Health Organization, Geneva.

[2] World Health Organization (2019) Global Tuberculosis Report 2019. World Health Organization, Geneva.

[3] World Health Organization (2017) Tuberculosis Country Brief, 2016. Armenia Vol 2017, World Health Organization, Geneva.

[4] Gebrekidan, G., Tesfaye, G., Hambisa, M.T. and Deyessa, N. (2015) Quality of Tuberculosis Care in Private Health Facilities of Addis Ababa, Ethiopia. Tuberculosis Research and Treatment, 2014, Article ID: 720432. https://doi.org/10.1155/2014/720432

[5] TB CARE I (2014) International Standards for Tuberculosis Care, Edition 3. TB CARE I, The Hague.

[6] Sadaphal, S., Kak, N., Holschneider, S., Smith-Artur, A. and Matji, R. (2013) Quality Improvement Handbook for TB and MDR-TB Programs. University Research CO., LLC, Chevy Chase.

[7] World Health Organization (2006) Quality of Care: A Process for Making Strategic Choices in Health Systems. World Health Organization, Geneva. 
[8] World Health Organization (2014) The End-TB Strategy. World Health Organization, Geneva.

[9] Khanal, S., Elsey, H., King, R., Baral, S.C., Bhatta, B.R. and Newell, J.N. (2017) Development of a Patient-Centred, Psychosocial Support Intervention for Multi-DrugResistant Tuberculosis (MDR-TB) Care in Nepal. PLoS ONE, 12, e0167559. https://doi.org/10.1371/journal.pone.0167559

[10] Nezenega, Z.S., Gacho, Y.H. and Tafere, T.E. (2013) Patient Satisfaction on Tuberculosis Treatment Service and Adherence to Treatment in Public Health Facilities of Sidama Zone, South Ethiopia. BMC Health Services Research, 110, Article No. 110. https://doi.org/10.1186/1472-6963-13-110

[11] O’Donnell, M.R., Daftary, A., Frick, M., Hirsch-Moverman, Y., Amico, K.R., Senthilingam, M., et al. (2016) Re-Inventing Adherence: Toward a Patient-Centered Model of Care for Drug-Resistant Tuberculosis and HIV. International Journal of Tuberculosis and Lung Disease, 20, 430-434. https://doi.org/10.5588/ijtld.15.0360

[12] Macq, J., Solis, A., Martinez, G. and Martiny, P. (2008) Tackling Tuberculosis Patients' Internalized Social Stigma through Patient Centred Care: An Intervention Study in Rural Nicaragua. BMC Public Health, 8, Article No. 154. https://doi.org/10.1186/1471-2458-8-154

[13] Reid, M.J.A. and Goosby, E. (2017) Patient-Centered Tuberculosis Programs Are Necessary to End the Epidemic. Journal of Infectious Diseases, 216, S673-S674. https://doi.org/10.1093/infdis/jix373

[14] World Health Organization (2017) A People-Centred Model of Tuberculosis Care: A Blueprint for Eastern European and Central Asian Countries. 1st Edition, World Health Organization Regional Office Europe, Copenhagen.

http://www.euro.who.int/ data/assets/pdf file/0004/342373/TB Content WHO P $\underline{\mathrm{RO}}$ eng final.pdf?ua $=1$

[15] Odone, A., Roberts, B., Dara, M., Van Den Boom, M., Kluge, H. and McKee, M. (2018) People- and Patient-Centred Care for Tuberculosis: Models of Care for Tuberculosis. International Journal of Tuberculosis and Lung Disease, 22, 133-138. https://doi.org/10.5588/ijtld.17.0608

[16] Khachadourian, V., Truzyan, N., Harutyunyan, A. et al. (2020) People-Centred Care versus Clinic-Based DOT for Continuation Phase TB Treatment in Armenia: A Cluster Randomized Trial. BMC Pulmonary Medicine, 20, Article No. 105. https://doi.org/10.1186/s12890-020-1141-y

[17] Khachadourian, V., Truzyan, N., Harutyunyan, A., Thompson, M.E., Harutyunyan, T. and Petrosyan, V. (2015) People-Centered Tuberculosis Care versus Standard Directly Observed Therapy: Study Protocol for a Cluster Randomized Controlled Trial. Trials, 16, Article No. 281. https://doi.org/10.1186/s13063-015-0802-2

[18] World Health Organization (2015) WHO Global Strategy on Integrated People-Centred Health Services 2016-2026: Executive Summary.

https://interprofessional.global/wp-content/uploads/2019/11/WHO-2015-Global-str ategy-on-integrated-people-centred-health-services-2016-2026.pdf

[19] Joint Commission International (2011) Joint Commission International Accreditation Standards for Hospitals. 4th Edition, Joint Commission International, Oakbrook Terrace.

[20] M'Imunya, J.M., Kredo, T. and Volmink, J. (2012) Patient Education and Counselling for Promoting Adherence to Treatment for Tuberculosis BT. Cochrane Database of Systematic Reviews, No. 5, Article No. CD006591.

[21] Truzyan, N., Crape, B., Harutyunyan, T. and Petrosyan, V. (2018) Family-Based Tuberculosis Counseling Supports Directly Observed Therapy in Armenia: A Pilot Project. 
Journal of Tuberculosis Research, 6, 113-124. https://doi.org/10.4236/jtr.2018.62011

[22] Groene, O. (2011) Patient Centredness and Quality Improvement Efforts in Hospitals: Rationale, Measurement, Implementation. International Journal for Quality in Health Care, 23, 531-537. https://doi.org/10.1093/intqhc/mzr058

[23] World Health Organization (2017) Ethics Guidance for the Implementation of the End Tb Strategy. World Health Organization, Geneva, 35-38. http://apps.who.int/iris/bitstream/10665/254820/1/9789241512114-eng.pdf?ua=1

[24] Leene, H.J.J. (1994) A Declaration on the Promotion of Patient' Rights in Europe. Tijdschrift voor Gezondheidsrecht, 18, Article No. 100. https://doi.org/10.1007/BF03055676

[25] Peled-Raz, M. (2017) Human Rights in Patient Care and Public Health-A Common Ground. Public Health Reviews, 38, Article No. 29. https://doi.org/10.1186/s40985-017-0075-2

[26] Truzyan, N., Grigoryan, Z., Musheghyan, L., Crape, B. and Petrosyan, V. (2019) Quality of Inpatient Tuberculosis Health Care in High-Burden Resource-Limited Settings: Protocol for a Comprehensive Mixed Methods Assessment Study. JMIR Research Protocols, 9, Article No. e13903. https://doi.org/10.2196/13903

[27] McCornack, L., Neal, C. and Triplett, J.L. (1994) The Qualitative Approach to Understanding Service Quality. Asia Pacific Journal of Marketing and Logistics, 6, 63-80. https://doi.org/10.1108/eb010257

[28] Meyer, J. (2000) Qualitative Research in Health Care: Using Qualitative Methods in Health Related Action Research. BMJ, 320, 178-181.

https://doi.org/10.1136/bmj.320.7228.178

[29] Curry, L.A., Nembhard, I.M. and Bradley, E.H. (2009) Qualitative and Mixed Methods Provide Unique Contributions to Outcomes Research. Circulation, 119, 14421452. https://doi.org/10.1161/CIRCULATIONAHA.107.742775

[30] Bradley, E.H., Curry, L.A. and Devers, K.J. (2007) Qualitative Data Analysis for Health Services Research: Developing Taxonomy, Themes, and Theory. Health Services Research, 42, 1758-1772. https://doi.org/10.1111/j.1475-6773.2006.00684.x

[31] Epstein, R.M., Fiscella, K., Lesser, C.S. and Stange, K.C. (2010) Analysis \& Commentary: Why the Nation Needs a Policy Push on Patient-Centered Health Care. Health Affairs, 29, 1489-1495. https://doi.org/10.1377/hlthaff.2009.0888

[32] Harutyunyan, T., Demirchyan, A., Thompson, M.E. and Petrosyan, V. (2010) Patient Satisfaction with Primary Care in Armenia: Good Rating of Bad Services? Health Services Management Research, 23, 12-17. https://doi.org/10.1258\%2Fhsmr.2009.009012

[33] Tekle, B., Mariam, D.H. and Ali, A. (2002) Defaulting from DOTS and Its Determinants in Three Districts of Arsi Zone in Ethiopia. International Journal of Tuberculosis and Lung Disease, 6, 573-579.

[34] Morisky, D.E., Malotte, Ck., Choi, P., Davidson, P., Rigler, S., Sugland, B., et al. (1990) A Patient Education Program to Improve Adherence Rates with Antituberculosis Drug Regimens. Health Education \& Behavior, 17, 253-266. https://doi.org/10.1177\%2F109019819001700303

[35] Ministry of Health (2020) Approval of the National Tuberculosis Treatment Guideline in Armenia. Order of the Minister of Health No. 2278-A. Ministry of Health, Yerevan. 